\title{
Long non-coding RNA LINC01627 is a prognostic risk factor for epithelial ovarian cancer
}

\author{
XIAOQING SHEN and WEIPEI ZHU \\ Department of Gynecology and Obstetrics, The Second Affiliated Hospital of Soochow University, \\ Suzhou, Jiangsu 215004, P.R. China
}

Received September 15, 2018; Accepted May 30, 2019

DOI: $10.3892 / \mathrm{ol} .2019 .10661$

\begin{abstract}
Ovarian malignancies are commonly diagnosed cancers of the female reproductive system. Recent studies have revealed that long non-coding RNAs (lncRNAs) can regulate a variety of oncological processes. In the present study, ovarian cancer expression datasets were searched in the GEO database using the GPL570 platform. Differential lncRNA expression between normal ovarian tissues and ovarian tumors were analyzed using the $\mathrm{R}$ package 'limma', and patient prognosis was accessed using the package 'survival'. Four databases, GSE14001, GSE18520, GSE38666 and GSE40595, were used for the analysis. A total of $64 \mathrm{lncRNAs}$ were highly expressed and 4 were downregulated within these four databases. Prognostic analysis of the $68 \mathrm{lncRNAs}$ in the four databases was performed, and revealed that the expression of long intergenic non-protein coding RNA 1627 (LINC01627) was negatively associated with patient prognosis in GSE19829 and GSE62193; there was no association between LINC01627 expression and patient's prognosis in GSE18520 or GSE63885. To investigate the proposed association between LINC01627 and patient prognosis, meta-analysis revealed that the total hazard ratio was 1.38 and the $95 \%$ confidence interval was between 1.04 and 1.83. Subgroup analysis revealed that LINC01627 may predict patient prognosis in high-grade, advanced and serous epithelial ovarian cancer, which was a risk factor for prognosis. Further assessment was performed in clinical samples and ovarian cancer cells, where the knockdown of LINC01627 inhibited the proliferative and migratory capacities of HO8910 and HEY cells. Collectively, the present results suggested that IncRNA LINC01627 may serve an oncogenic role in the development of epithelial ovarian tumors.
\end{abstract}

Correspondence to: Dr Weipei Zhu, Department of Gynecology and Obstetrics, The Second Affiliated Hospital of Soochow University, 1055 Sanxiang Road, Suzhou, Jiangsu 215004, P.R. China E-mail: 18262633089@163.com

Key words: long non-coding RNA, LINC01627, epithelial ovarian cancer, prognosis

\section{Introduction}

Ovarian cancer has the highest mortality rate of all gynecological malignancies, and epithelial ovarian cancer (EOC) is the most frequently encountered (1). The disease course of ovarian cancer is complex, and symptoms are non-specific (2). Therefore, at the point of diagnosis the majority of patients have reached the advanced stages of disease, when radical treatment is less successful (3). Current issues in the diagnosis and treatment of ovarian cancer include a lack of early diagnosis, limited effective treatment options, and propensity to chemoresistance and metastatic dissemination $(4,5)$. Therefore, the discovery of novel diagnostic markers and therapeutic targets is pivotal to improving the prognosis for patients with EOC.

Long non-coding RNAs (lncRNAs) are a type of non-coding RNA $>200$ nucleotides in length, known as the 'dark matter' of the genome (6). Although they are not protein-coding, IncRNAs possess complex biological functions $(7,8)$. Yan et al (9) analyzed the expression of lncRNAs in 5,037 human tissue samples from The Cancer Genome Atlas (TCGA) database, across 13 types of cancer. It was revealed that, in different types of cancer tissue, non-coding genes had a specific expression profile compared with their protein-coding counterparts. Therefore, the study of IncRNAs may hold promise towards improving cancer diagnosis and treatment programs (10-12). However, to the best of our knowledge, there is currently limited research into the expression and function of IncRNAs in EOC. Therefore, the present study aimed to improve the understanding of the functions and molecular mechanisms of lncRNAs in EOC, and how they may contribute to the early diagnosis and treatment of ovarian cancer $(13,14)$.

In previous years, the development of chip technology has greatly enhanced the efficiency of biological research (15). Bioinformatics is an interdisciplinary field of biology and computer science, and with the advent of 'big data' in health care, bioinformatics research has greatly improved the efficiency and progress of health care-associated research (16).

\section{Materials and methods}

Data processing. Ovarian cancer expression files GSE18520(17),GSE19829(18),GSE26193(19),GSE63885(20), GSE14001 (21), GSE38666 (22) and GSE40595 (23) were 
retrieved from the Gene Expression Omnibus (GEO) database (platform GPL570). Datasets involving patients with EOC were selected; the differential expression levels of lncRNAs between normal ovarian and ovarian tumor tissues were analyzed using the R (3.3.3) package 'limma' (3.40.2)' (http://bioconductor.org/packages/release/bioc/html/limma. $\mathrm{html}$ ), and disease prognosis was analyzed using the $\mathrm{R}$ package 'survival'. The low grades included grade 1 and 2 and the high grade included grade 3 . According to the International Federation of Gynaecology and Obstetrics (FIGO) staging systems, stages III and IV included advanced stages and stages I and II included early stage. The median expression of LINC01627 was used as cut-off value for survival analysis. Where $\mathrm{P} \geq 0.1$ and $\mathrm{I}^{2} \leq 50 \%$ indicated no heterogeneity between each study, meta-analysis was performed using the fixed effect model. Where $\mathrm{P}<0.1$ and $\mathrm{I}^{2}>50 \%$ suggested heterogeneity between each study, the random effects model was used for meta-analysis, and sub-analysis was conducted to determine the source of heterogeneity.

Tissues sample collection and total RNA extraction. A total of 20 EOC tissue samples and 10 normal ovarian tissue samples were collected from patients who underwent surgery between June 2015 and October 2018 at the Second Affiliated Hospital of Soochow University (Suzhou, China). The present study was approved by the Ethics Committee of the Second Affiliated Hospital of Soochow University (Suzhou, China), and all patients provided written informed consent. None of the patients had received chemotherapy or radiotherapy prior to surgery and all patients were treated with systemic platinum-based chemotherapy following surgery. Tumor stage was determined according to the FIGO staging system. Following resection, the tissues were immediately frozen at $-80^{\circ} \mathrm{C}$. TRIzol ${ }^{\circledR}$ reagent (Invitrogen; Thermo Fisher Scientific, Inc.) was used to extract the total RNA from ovarian cancer cells and tissue samples.

Reverse transcription-quantitative $P C R$ (RT-qPCR). Total RNA was reverse transcribed into cDNA using the PrimeScript $^{\mathrm{TM}}$ RT Master Mix (cat. no. RR036A; Clontech Laboratories, Inc.), and qPCR was performed using the $\mathrm{SYBR}^{\circledR}$ Green Master Mix (Tiangen Biotech Co., Ltd.), according the manufacturer's protocol. The reaction volume total was $10 \mu \mathrm{l}$, and the thermocycling conditions were: $95^{\circ} \mathrm{C}$ pre-denaturation for $3 \mathrm{sec}$, followed by 40 cycles of $95^{\circ} \mathrm{C}$ denaturation for $10 \mathrm{~min}, 60^{\circ} \mathrm{C}$ annealing for $40 \mathrm{sec}$, followed by $72^{\circ} \mathrm{C}$ extension for $30 \mathrm{sec}$. The $2^{-\Delta \Delta \mathrm{Cq}}$ method was used to calculate relative expression levels (24). Primers were as follows: LINC01627, forward 5'-ACTGATACCCACvACAGAAGAAG-3, and reverse 5'-CCCGTCAAGTCGAGTAATATCC-3'; and GAPDH, forward 5'-GCACCGTCAAGGCTGAGAAC-3', and reverse 5'-GGATCTCGCTCCTGGAAGATG-3'.

Cell culture and transfection. Ovarian cancer cell lines HO8910 and HEY were purchased from the American Type Culture Collection. The ovarian cancer cell lines HO8910 and HEY were cultured in RPMI 1640 (Gibco; Thermo Fisher Scientific, Inc.) containing 10\% fetal bovine serum (FBS; cat. no. 10099-141; Gibco; Thermo Fisher Scientific, Inc.) at $37^{\circ} \mathrm{C}$ with $5 \% \mathrm{CO}_{2}$. Cells were seeded in 6-well plates and once reached $70 \%$ confluency, cells were transfected with $50 \mathrm{nM}$ negative control (NC) or LINC01627 small interfering (si)RNA using Lipofectamine ${ }^{\mathrm{TM}}$ RNAi MAX reagent (Thermo Fisher Scientific, Inc.), according to the manufacturer's protocol. The LINC01627 siRNA sequence was 5'-GGGTGTACACAGAGT ATAA-3'. The negative control sequence was 5'-UUCUCC GAACGUGUCACGUTT-3'. After $24 \mathrm{~h}$ of transfection, subsequent experiments were performed.

Cell migration assay. Following transfection, cells were resuspended in RPMI medium without FBS at a density of $2 \times 10^{4}$ cells/well. A total of $100 \mu 1$ resuspended cells were seeded into the upper chamber of a Transwell insert. RPMI medium supplemented with $10 \%$ FBS was added to the lower chamber. The cells were cultured at $37^{\circ} \mathrm{C}$ with $5 \% \mathrm{CO}_{2}$ for $25 \mathrm{~h}$. Migratory cells were fixed in $70 \%$ paraformaldehyde for $30 \mathrm{~min}$ and stained with $0.1 \%$ crystal violet for a further $30 \mathrm{~min}$ at room temperature. Following washing with PBS, cells were directly observed using an inverted microscope and was counted in five randomly selected fields to get the average using ImageJ (version 1.48; National Institutes of Health).

Cell proliferation assay. Following transfection, HO8910 and HEY cells were seeded into 96-well plates at a density of $5 \times 10^{3}$ cells/well. At 24, 48, 72 and $96 \mathrm{~h}$ time points, $10 \mu \mathrm{l}$ Cell Counting Kit-8 (CCK-8; Dojindo Molecular Technologies, Inc.) solution was added to each well and the plates were incubated at $37^{\circ} \mathrm{C}$ for $1 \mathrm{~h}$ in the dark. Absorbance was measured at a wavelength of $450 \mathrm{~nm}$ using a microplate reader.

Statistical analysis. Stata statistical software (StataCorp LP) was used to perform statistical analyses. The hazard ratio (HR) and 95\% confidence interval (CI) were determined using the log-rank test. Unpaired t-test was enrolled to compute the difference between two groups. $\mathrm{P}<0.05$ was considered to indicate a statistically significant difference. Each experiment was repeated three times.

\section{Results}

LINC01627 is highly expressed in ovarian cancer and is a prognostic risk factor for EOC. In order to determine potential prognostic factors for ovarian cancer, a group of differentially expressed genes was obtained from the GSE14001, GSE18520, GSE38666 and GSE40595 databases using the R package 'limma' (Fig. 1A). Using the R package 'venn', a total of 64 lncRNAs were identified as highly expressed in GSE14001, GSE18520, GSE38666 and GSE4059 (Fig. 1B), and 4 were downregulated (Fig. 1C). Prognostic analysis of the 64 lncRNAs was performed in the GSE18520, GSE19829, GSE62193 and GSE63885 databases. The results revealed that only LINC01627 expression was negatively correlated with patient outcome in GSE19829 and GSE62193 (Fig. 2B and C), whilst no association was observed in GSE18520 or GSE63885 (Fig. 2A and D). To investigate whether LINC01627 was associated with patient prognosis, a total HR (1.38) interval of 1.04-1.83 was determined using meta-analysis (Fig. 2E), suggesting that LINC01627 may be a prognostic factor in predicting patient outcome. 
A

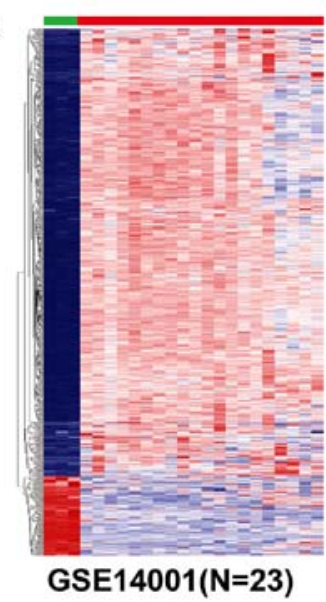

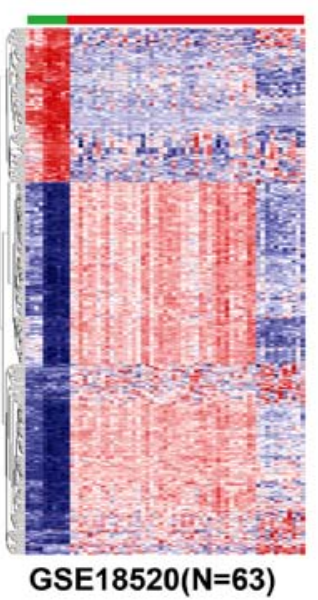

B

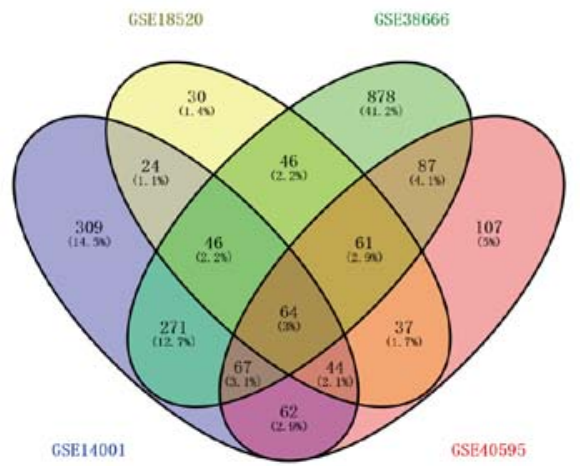

Upregulated
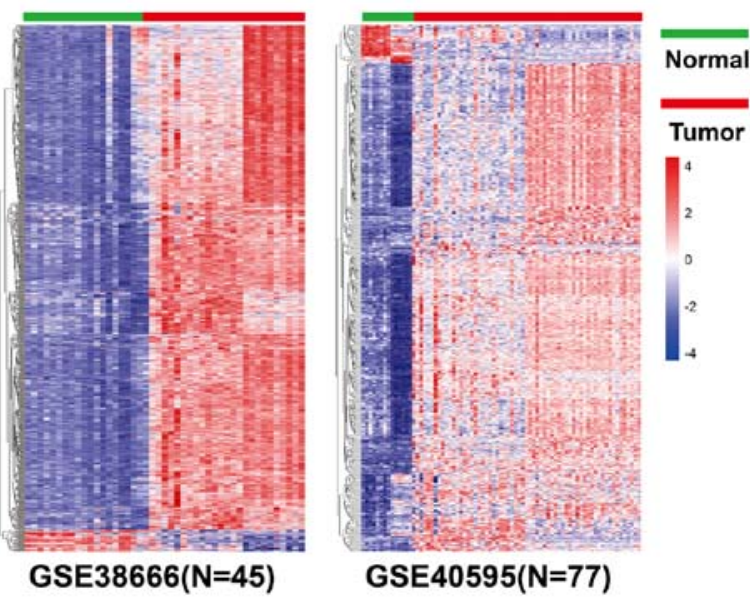

C
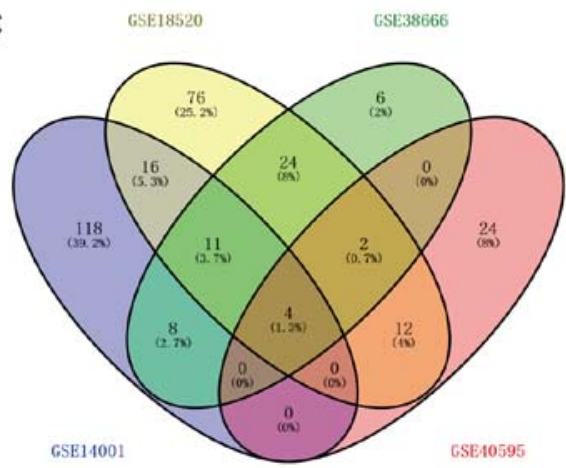

Downregulated

Figure 1. Differentially expressed lncRNAs in ovarian cancer. (A) Heat maps of dysregulated lncRNAs in GSE14001, GSE18520, GSE38666 and GSE40595 databases. (B) Venn diagram of upregulated lncRNAs shows 64 lncRNAs in common in the 4 datasets. (C) Venn diagram of downregulated lncRNAs shows 4 lncRNAs in common in the 4 datasets. Red represents upregulated genes and blue represents downregulated genes. IncRNAs, long non-coding RNAs.
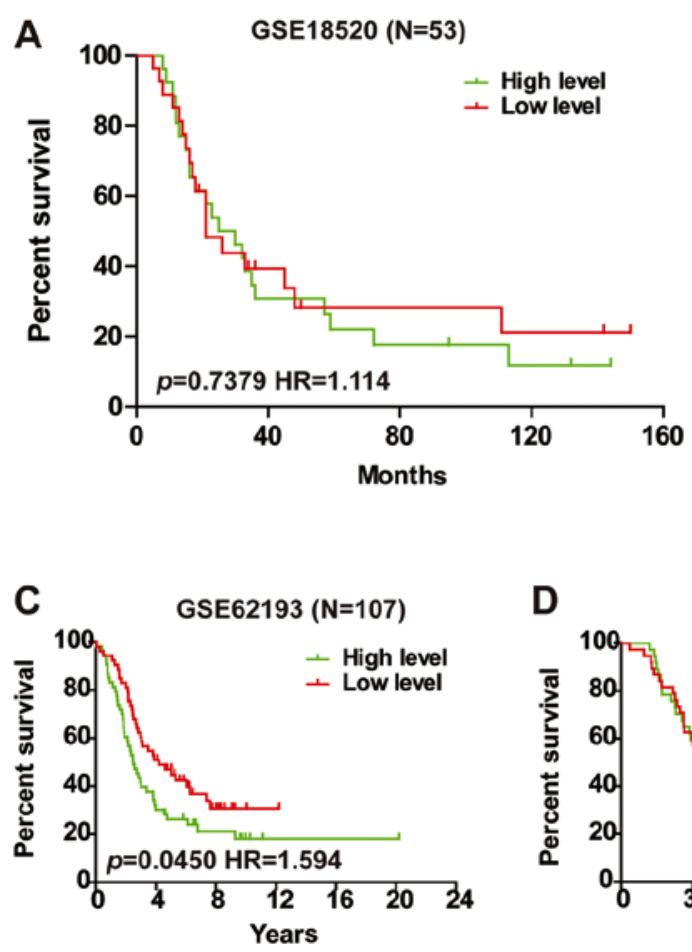

B

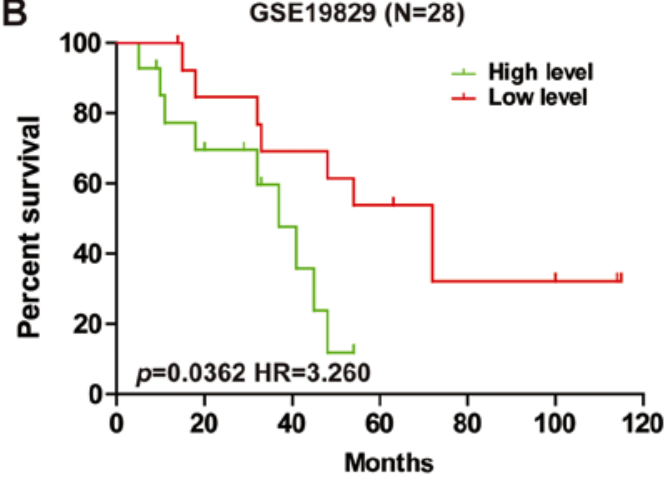

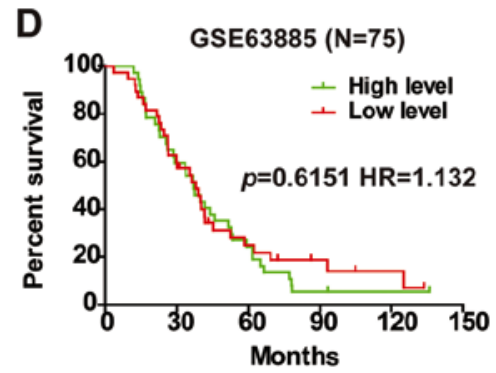

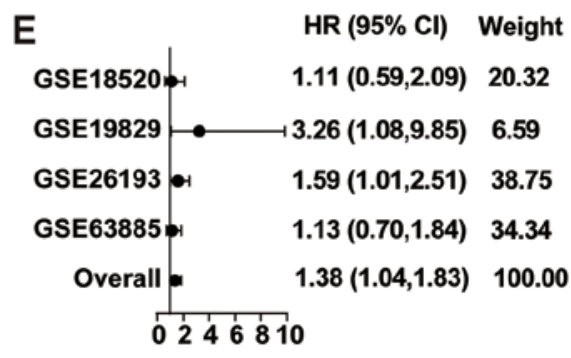

Figure 2. LINC01627 is a prognostic factor in EOC. Kaplan-Meir plots showing the association of LINC01627 expression with patient survival in the (A) GSE18520, (B) GSE19829, (C) GSE62193 and (D) GSE63885 datasets. (E) Meta-analysis revealed that LINC01627 was negatively correlated with the outcome of patients with EOC. CI, confidence interval; EOC, epithelial ovarian cancer; HR, hazard ratio; LINC01627, long intergenic non-protein coding RNA 1627. 

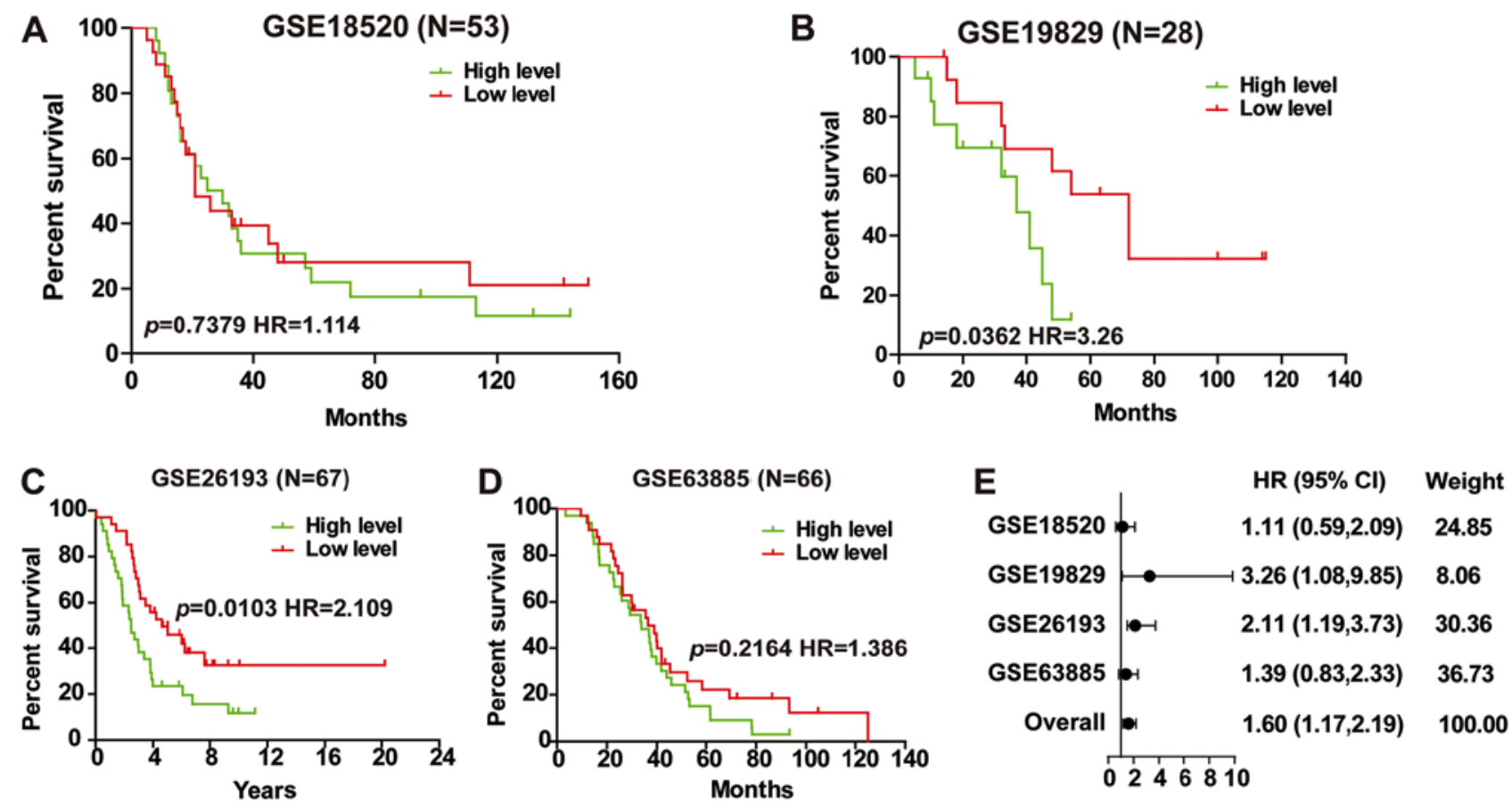

Figure 3. LINC01627 is a prognostic risk factor for high-grade EOC. Kaplan-Meir plots showing the association of LINC01627 expression with patient survival in the (A) GSE18520, (B) GSE19829, (C) GSE62193 and (D) GSE63885 datasets. (E) Meta-analysis revealed that LINC01627 was negatively correlated with the outcome of patients with high-grade EOC. CI, confidence interval; EOC, epithelial ovarian cancer; HR, hazard ratio; LINC01627, long intergenic non-protein coding RNA 1627.
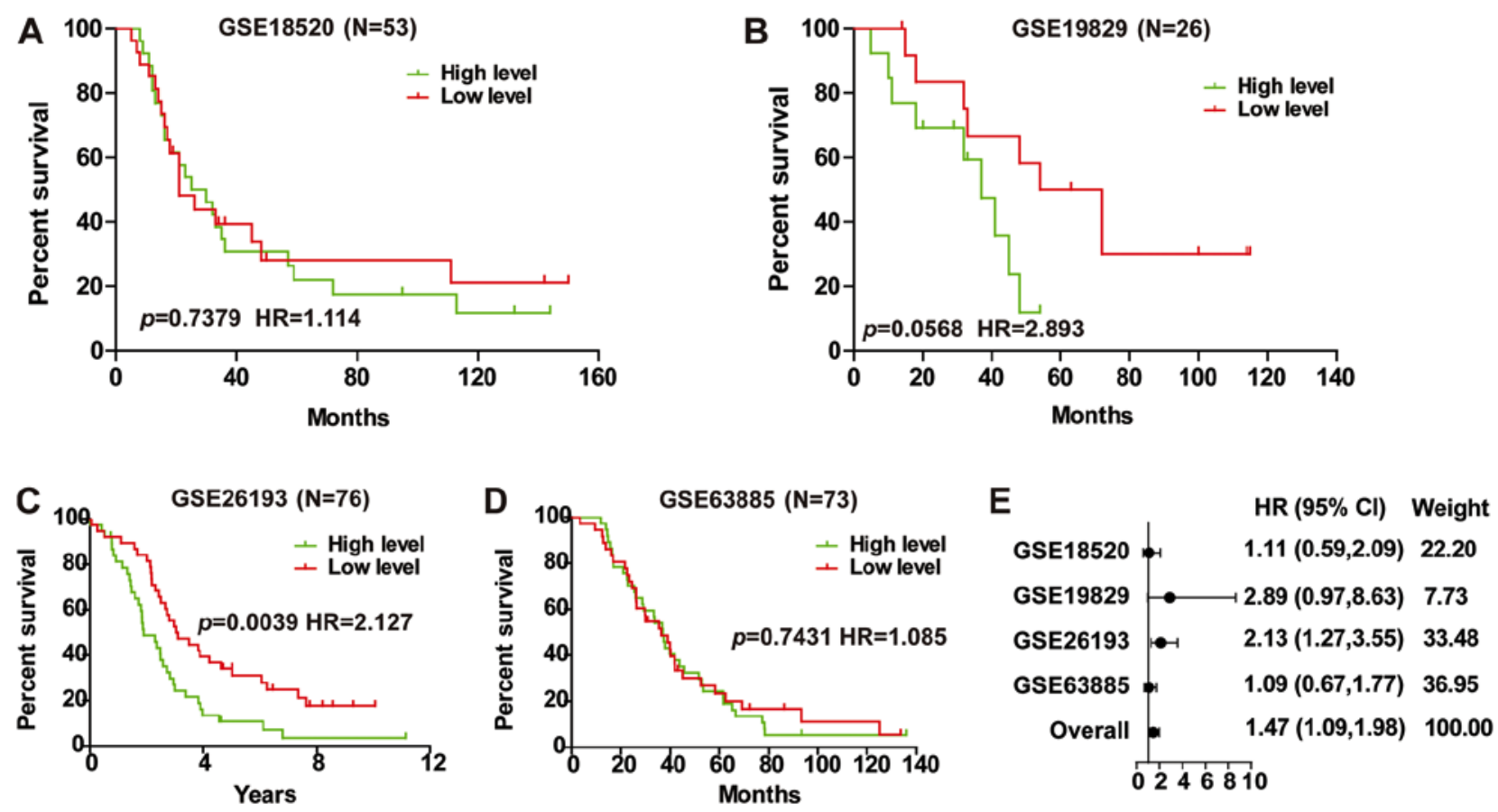

Figure 4. LINC01627 is a prognostic risk factor for advanced EOC. Kaplan-Meir plots showing the association of LINC01627 expression with patient survival in the (A) GSE18520, (B) GSE19829, (C) GSE62193 and (D) GSE63885 datasets. (E) Meta-analysis revealed that LINC01627 was negatively correlated with the outcome of patients with advanced EOC. CI, confidence interval; EOC, epithelial ovarian cancer; HR, hazard ratio; LINC01627, long intergenic non-protein coding RNA 1627.

LINC01627 is a prognostic risk factor for high-grade $E O C$. Further analysis of the prognostic implications of LINC01627 in high-grade EOC illustrated that the expression of LINC01627 was negatively correlated with patient prognosis in GSE19829 and GSE26193 (Fig. 3B and C), whilst no correlation was observed in GSE18520 or GSE63885 (Fig. 3A and D). A total HR (1.60) interval of 1.17-2.19 was determined using meta-analysis (Fig. 3E), suggesting that LINC01627 may be regarded as a prognostic factor for high-grade EOC. 

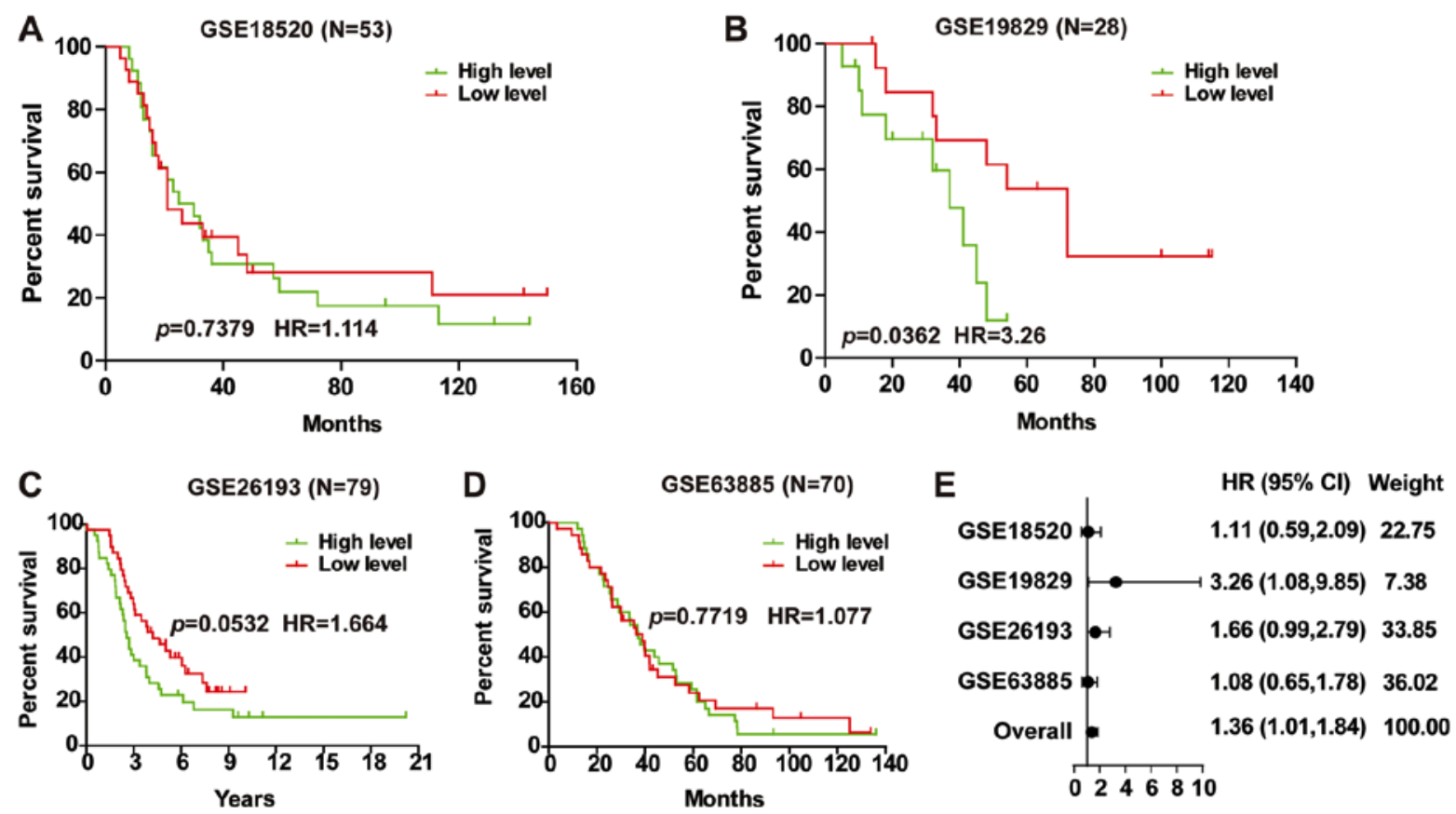

Figure 5. LINC01627 is a prognostic risk factor for serous EOC. Kaplan-Meir plots showing the association of LINC01627 expression with patient survival in the (A) GSE18520, (B) GSE19829, (C) GSE62193 and (D) GSE63885 datasets. (E) Meta-analysis revealed that LINC01627 was negatively correlated with the outcome of patients with serous EOC. CI, confidence interval; EOC, epithelial ovarian cancer; HR, hazard ratio; LINC01627, long intergenic non-protein coding RNA 1627.
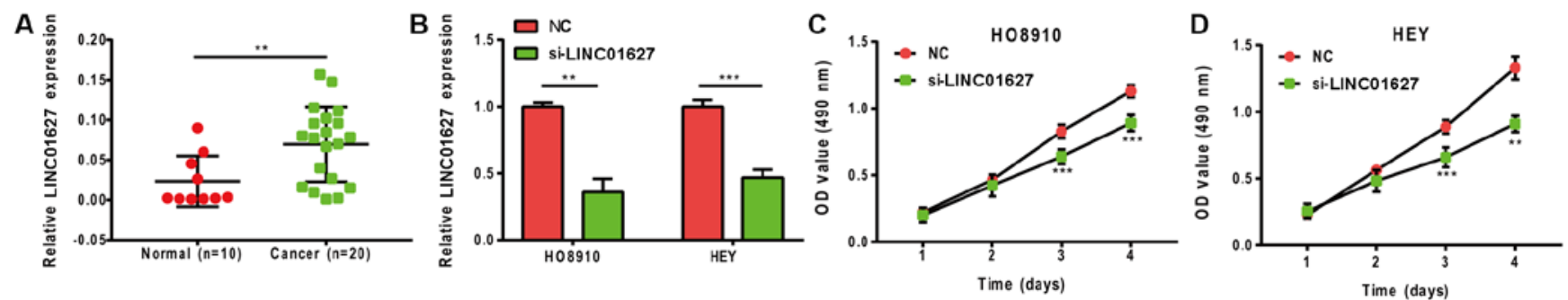

E

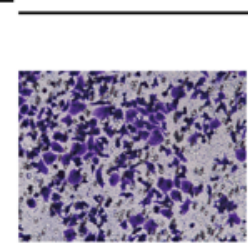

NC
H08910

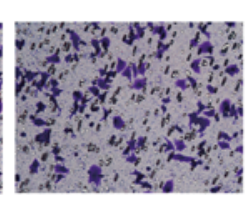

Si-LINC01627

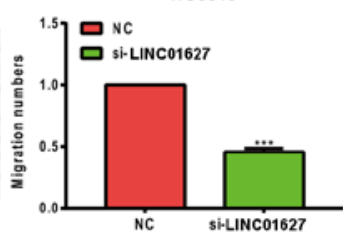

F

HEY

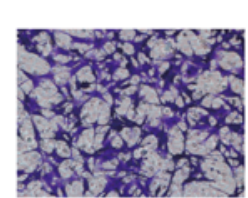

NC

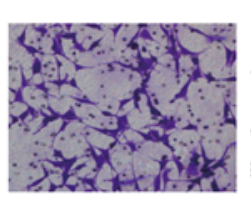

Si-LINC01627

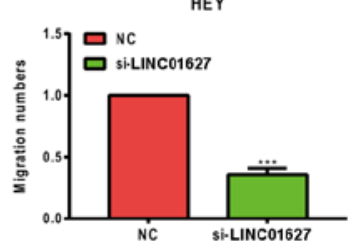

Figure 6. LINC01627 promotes proliferation and migration in ovarian cancer cells in vitro. (A) LINC01627 levels were higher in ovarian cancer samples compared with normal ovarian tissues. (B) siRNA transfection efficiency. (C) Cell proliferation was measured by Cell Counting Kit-8 assay in HO8910 and (D) HEY cells following LINC01627 silencing. (E) Cell migration was measured by Transwell assay in HO8910 and (F) HEY cells following LINC01627 silencing (magnification, $\mathrm{x} 200$ ). siRNA, small interfering RNA. ${ }^{* *} \mathrm{P}<0.01$ and ${ }^{* * *} \mathrm{P}<0.001$ compared with NC group or Normal. C, negative control; LINC01627, long intergenic non-protein coding RNA 1627; si, small interfering.

LINC01627 is a prognostic risk factor for advanced EOC. Investigation into the prognostic implications of LINC01627 in advanced EOC demonstrated that LINC01627 was a prognostic risk factor within GSE26193 (Fig. 4C), whilst no correlation was revealed in GSE18520, GSE19829 or GSE63885 (Fig. 4A, $\mathrm{B}$ and D). To investigate whether LINC01627 was associated with the prognosis of patients with advanced EOC, a total HR (1.47) interval of 1.09-1.98 was determined using meta-analysis (Fig. 4E), suggesting that LINC01627 may be regarded as a prognostic factor for advanced EOC.
LINC01627 is a prognostic risk factor for serous EOC. EOC includes serous, mucinous, endometrioid and other subtypes, of which serous EOC is the most frequently observed (25). Analysis of the prognostic implication of LINC01627 in serous EOC illustrated that LINC01627 was a prognostic risk factor within GSE19829 (Fig. 5B), whilst no correlation was observed in GSE18520, GSE26193 or GSE63885 (Fig. 5A, C and D). To investigate whether LINC01627 is associated with the prognosis of patients with serous EOC, a total HR (1.36) interval 1.01-1.84 was determined using meta-analysis 
(Fig. 5E), suggesting that LINC01627 may be regarded as a prognostic factor for serous EOC. In addition, it was indicated that increased expression of LINC01627 was associated with poor patient prognosis.

LINC01627 promotes proliferation and migration in ovarian cancer cells. LINC01627 expression levels were detected in normal ovarian $(n=10)$ and EOC tissues $(n=20)$. The results revealed that the expression levels of LINC01627 were significantly increased in EOC tissues ( $\mathrm{P}=0.0090$; Fig. 6A). To further investigate the functions of LINC01627, siRNA targeting LINC01627 was established and knockdown assays were subsequently performed in vitro. Transfection efficiency is illustrated in Fig. 6B, with the si-LINC01627 group exhibiting significantly decreased levels of LINC01627 compared with the control group, in both cell lines tested. The results of the CCK- 8 assay revealed that cell proliferation was significantly inhibited following LINC01627-knockdown (Fig. 6C and D). In addition, results from Transwell assays demonstrated that migration was significantly abrogated in HO8910 and HEY cells following LINC01627 knockdown (Fig. 6E and F).

\section{Discussion}

With limited early clinical symptoms and the current limitations of medical treatments, including drug resistance, the majority of patients with ovarian cancer are in the advanced stages of disease at the time of diagnosis (26). Although cytoreductive surgery combined with paclitaxel and cisplatin chemotherapy is able to effectively relieve symptoms in the majority of patients, the 5-year survival rate of patients with ovarian epithelial tumors is $<40 \%(27,28)$. At present, the most frequently used diagnostic methods for ovarian cancer include ultrasound and the detection of cancer antigen 125 and human epididymal protein 4 (29), the specificity and sensitivity of which do not meet the requirements for early screening. As a result, the early detection and diagnosis of ovarian cancer has yet to be optimized. To improve the survival rates of patients with EOC novel tumor biomarkers and therapeutic targets are required (30).

LncRNAs were discovered in the 1980s and due to their inability to encode proteins, they were previously considered to be 'transcription noise' (6). In recent years, an increasing number of studies have indicated that lncRNAs are able to regulate gene expression at the epigenetic, transcriptional and post-transcriptional levels (31). In addition, they are likely to serve a prominent role in the development of cancer (32). It has been reported that there are significant differences in the expression levels of lncRNAs between healthy and tumorous tissues, as brain cytoplasmic RNA 1 (BCYRN1) was highly expressed in breast, lung, tongue and ovarian cancer (33), and HOX transcript antisense RNA (HOTAIR) expression levels were significantly higher in tumor tissues compared with those in normal, healthy tissues (34-36). Therefore, lncRNAs are potential novel tumor markers and therapeutic targets for the screening and early diagnosis of cancer (37-39). The mechanisms of lncRNAs in the development of ovarian cancer may also be implicated in the prevention, diagnosis, treatment and prognosis of the disease $(40,41)$.
The aim of the present study was to determine the role of lncRNAs in the development of EOC, to analyse the expression of LINC01627 in different databases, and to conduct meta-analysis in order to confirm whether LINC01627 was a prognostic factor for EOC. The results revealed that LINC01627 was a prognostic risk factor for EOC. Furthermore, meta-analysis concluded that LINC01627 was a prognostic risk factor for high-grade, advanced and serous EOC, as the higher the expression, the poorer the patient prognosis. It was also concluded that LINC01627 expression levels may have prognostic significance in patients with EOC. Upregulation of LINC01627 has been observed in non-small-cell lung cancer (42); however, the role of LINC01627 in EOC is yet to be ascertained. In the present study, to further investigate the oncogenic role of LINC01627, the expression levels of LINC01627 were determined in EOC clinical samples using RT-qPCR. The results revealed that LINC01627 was highly expressed in EOC tissues compared with normal ovarian tissue samples. Functional cell-based assays further confirmed that LINC01627 had a role on the proliferation and migration of HO8910 and HEY cells. To further examine the mechanism of LINC01627, RNA immunoprecipitation and chromatin immunoprecipitation assays are going to be performed in future studies.

With further research, LINC01627 may become a future therapeutic target for the treatment of ovarian cancer, which may accelerate the use of 1 ncRNAs from basic research to clinical application, and provide a novel opportunity for the diagnosis and treatment of ovarian cancer. These results may subsequently promote novel ideas for the research, diagnosis and treatment of EOC. However, since the investigation of lncRNAs is a relatively new research field, expression profiles in EOC have yet to be fully established. The understanding of the molecular mechanisms of lncRNAs is limited and further investigation is required in order to examine their full role and potential in the development of EOC. In conclusion, the present study indicated that LINC01627 may be a prognostic risk factor for EOC, and may predict patient prognosis in high-grade, advanced and serous disease subtypes.

\section{Acknowledgements}

The authors would like to thank Nanjing Qiaoyuan Biotechnology Co., Ltd for their help with bioinformatics analysis.

\section{Funding}

The present study was funded by The Jiangsu Maternal and Child Healthcare Research Project (grant no. F201709).

\section{Availability of data and materials}

The data used to support the findings of this study are available from the corresponding author upon request.

\section{Authors' contributions}

XS performed most experiments, collected clinical tissues and analysed data. WZ designed the project and edited the manuscript. 


\section{Ethics approval and consent to participate}

The present study was approved by the Ethics Committee of the Second Affiliated Hospital of Soochow University (Suzhou, China), and all patients provided written informed consent.

\section{Patient consent for publication}

Not applicable.

\section{Competing interests}

The authors declare that they have no competing interests.

\section{References}

1. Meinsohn MC, Smith OE, Bertolin K and Murphy BD: The orphan nuclear receptors steroidogenic factor-1 and liver receptor homolog-1: Structure, regulation, and essential roles in mammalian reproduction. Physiol Rev 99: 1249-1279, 2019.

2. George A, Kaye S and Banerjee S: Delivering widespread BRCA testing and PARP inhibition to patients with ovarian cancer. Nat Rev Clin Oncol 14: 284-296, 2017.

3. Tung N, Domchek SM, Stadler Z, Nathanson KL, Couch F, Garber JE, Offit K and Robson ME: Counselling framework for moderate-penetrance cancer-susceptibility mutations. Nat Rev Clin Oncol 13: 581-588, 2016.

4. Ren C, Li X, Wang T, Wang G, Zhao C, Liang T, Zhu Y, Li M, Yang C, Zhao $\mathrm{Y}$ and Zhang GM: Functions and mechanisms of long noncoding RNAs in ovarian cancer. Int J Gynecol Cancer 25: 566-569, 2015.

5. Zhang LQ, Yang SQ, Wang Y, Fang Q, Chen XJ, Lu HS and Zhao LP: Long noncoding RNA MIR4697HG promotes cell growth and metastasis in human ovarian cancer. Anal Cell Pathol (Amst) 2017: 8267863, 2017.

6. Esposito R, Bosch N, Lanzos A, Polidori T, Pulido-Quetglas C and Johnson R: Hacking the cancer genome: Profiling therapeutically actionable long non-coding RNAs using CRISPR-Cas9 screening. Cancer Cell 35: 545-557, 2019.

7. Spizzo R, Almeida MI, Colombatti A and Calin GA: Long non-coding RNAs and cancer: A new frontier of translational research? Oncogene 31: 4577-4587, 2012.

8. Chu C, Spitale RC and Chang HY: Technologies to probe functions and mechanisms of long noncoding RNAs. Nat Struct Mol Biol 22: 29-35, 2015

9. Yan X, Hu Z, Feng Y, Hu X, Yuan J, Zhao SD, Zhang Y, Yang L, Shan W, He Q, et al: Comprehensive genomic characterization of long non-coding RNAs across human cancers. Cancer Cell 28: 529-540, 2015

10. Bartonicek N, Maag JL and Dinger ME: Long noncoding RNAs in cancer: Mechanisms of action and technological advancements. Mol Cancer 15: 43, 2016.

11. Li T, Yang XD, Ye CX, Shen ZL, Yang Y, Wang B, Guo P, Gao ZD, Ye YJ, Jiang KW and Wang S: Long noncoding RNA HIT000218960 promotes papillary thyroid cancer oncogenesis and tumor progression by upregulating the expression of high mobility group AT-hook 2 (HMGA2) gene. Cell Cycle 16: 224-231, 2017

12. Peng L, Yuan XQ, Liu ZY, Li WL, Zhang CY, Zhang YQ, Pan X, Chen J, Li YH and Li GC: High lncRNA H19 expression as prognostic indicator: Data mining in female cancers and polling analysis in non-female cancers. Oncotarget 8: 1655-1667, 2017.

13. Jiang $\mathrm{C}$, Li X, Zhao $\mathrm{H}$ and Liu H: Long non-coding RNAs: Potential new biomarkers for predicting tumor invasion and metastasis. Mol Cancer 15: 62, 2016.

14. Meryet-Figuière M,Lambert B, Gauduchon P, Vigneron N, Brotin E, Poulain L and Denoyelle C: An overview of long non-coding RNAs in ovarian cancers. Oncotarget 7: 44719-44734, 2016.

15. Baumgart S, Hölters S, Ohlmann CH, Bohle R, Stöckle M, Ostenfeld MS, Dyrskjøt L, Junker K and Heinzelmann J: Exosomes of invasive urothelial carcinoma cells are characterized by a specific miRNA expression signature. Oncotarget 8 : 58278-58291, 2017.
16. Lo CM, Iqbal U and Jack Li YC: Automatic methods for managements of cancer, medicine, and behavior. Comput Methods Programs Biomed 146: A1, 2017.

17. Mok SC, Bonome T, Vathipadiekal V, Bell A, Johnson ME, Wong KK, Park DC, Hao K, Yip DK, Donninger H, et al: A gene signature predictive for outcome in advanced ovarian cancer identifies a survival factor: Microfibril-associated glycoprotein 2. Cancer Cell 16: 521-532, 2009.

18. Konstantinopoulos PA, Spentzos D, Karlan BY, Taniguchi T, Fountzilas E, Francoeur N, Levine DA and Cannistra SA: Gene expression profile of BRCAness that correlates with responsiveness to chemotherapy and with outcome in patients with epithelial ovarian cancer. J Clin Oncol 28: 3555-3561, 2010.

19. Gentric G, Kieffer Y, Mieulet V, Goundiam O, Bonneau C, Nemati F, Hurbain I, Raposo G, Popova T, Stern MH, et al: PML-regulated mitochondrial metabolism enhances chemosensitivity in human ovarian cancers. Cell Metab 29: 156-173 e10, 2019.

20. Lisowska KM, Olbryt M, Dudaladava V, Pamuła-Piłat J, Kujawa K, Grzybowska E, Jarząb M, Student S, Rzepecka IK, Jarząb B and Kupryjańczyk J: Gene expression analysis in ovarian cancer-faults and hints from DNA microarray study. Front Oncol 4: 6, 2014.

21. Tung CS, Mok SC, Tsang YT, Zu Z, Song H, Liu J, Deavers MT, Malpica A, Wolf JK, Lu KH, et al: PAX2 expression in low malignant potential ovarian tumors and low-grade ovarian serous carcinomas. Mod Pathol 22: 1243-1250, 2009.

22. Lili LN, Matyunina LV, Walker LD, Benigno BB and McDonald JF: Molecular profiling predicts the existence of two functionally distinct classes of ovarian cancer stroma. Biomed Res Int 2013: 846387, 2013.

23. Yeung TL, Leung CS, Wong KK, Samimi G, Thompson MS, Liu J, Zaid TM, Ghosh S, Birrer MJ and Mok SC: TGF- $\beta$ modulates ovarian cancer invasion by upregulating $\mathrm{CAF}$-derived versican in the tumor microenvironment. Cancer Res 73: 5016-5028, 2013.

24. Livak KJ and Schmittgen TD: Analysis of relative gene expression data using real-time quantitative PCR and the 2(-Delta Delta $\mathrm{C}(\mathrm{T})$ ) method. Methods 25: 402-408, 2001.

25. Erol A, Niemira M and Kretowski AJ: Novel approaches in ovarian cancer research against heterogeneity, late diagnosis, drug resistance, and transcoelomic metastases. Int J Mol Sci 20: pii: E2649, 2019.

26. Franier B and Thompson M: Early stage detection and screening of ovarian cancer: A research opportunity and significant challenge for biosensor technology. Biosens Bioelectron 135: 71-81, 2019.

27. Oberaigner $\mathrm{W}$, Minicozzi P, Bielska-Lasota M, Allemani C, de Angelis R, Mangone L and Sant M; Eurocare Working Group: Survival for ovarian cancer in Europe: The across-country variation did not shrink in the past decade. Acta Oncol 51: 441-453, 2012.

28. Shapira I, Oswald M, Lovecchio J, Khalili H, Menzin A, Whyte J, Dos Santos L, Liang S, Bhuiya T, Keogh M, et al: Circulating biomarkers for detection of ovarian cancer and predicting cancer outcomes. Br J Cancer 110: 976-983, 2014.

29. Dochez V, Caillon H, Vaucel E, Dimet J, Winer $\mathrm{N}$ and Ducarme G: Biomarkers and algorithms for diagnosis of ovarian cancer: CA125, HE4, RMI and ROMA, a review. J Ovarian Res 12: 28, 2019.

30. Li K, Hüsing A, Fortner RT, Tjønneland A, Hansen L, Dossus L, Chang-Claude J, Bergmann M, Steffen A, Bamia C, et al: An epidemiologic risk prediction model for ovarian cancer in Europe: The EPIC study. Br J Cancer 112: 1257-1265, 2015.

31. Qin Y, Liu X, Pan L, Zhou R and Zhang X: Long noncoding RNA MIR155HG facilitates pancreatic cancer progression through negative regulation of miR-802. J Cell Biochem: Jun 3, 2019 (Epub ahead of print)

32. Huang Z, Luo Q, Yao F, Qing C, Ye J, Deng Y and Li J: Identification of differentially expressed long non-coding RNAs in polarized macrophages. Sci Rep 6: 19705, 2016.

33. Hu T and Lu YR: BCYRN1, a c-MYC-activated long non-coding RNA, regulates cell metastasis of non-small-cell lung cancer. Cancer Cell Int 15: 36, 2015.

34. Martínez-Fernández M, Feber A, Dueñas M, Segovia C, Rubio C, Fernandez M, Villacampa F, Duarte J, López-Calderón FF, Gómez-Rodriguez MJ, et al: Analysis of the Polycomb-related lncRNAs HOTAIR and ANRIL in bladder cancer. Clin Epigenetics 7: 109, 2015. 
35. Zhang A, Zhao JC, Kim J, Fong KW, Yang YA, Chakravarti D, Mo YY and Yu J: LncRNA hotair enhances the androgen-receptor-mediated transcriptional program and drives castration-resistant prostate cancer. Cell Rep 13: 209-221, 2015.

36. Yang XD, Xu HT, Xu XH, Ru G, Liu W, Zhu JJ, Wu YY, Zhao K, Wu Y, Xing CG, et al: Knockdown of long non-coding RNA HOTAIR inhibits proliferation and invasiveness and improves radiosensitivity in colorectal cancer. Oncol Rep 35: 479-487, 2016.

37. Bolha L, Ravnik-Glavač M and Glavač D: Long noncoding RNAs as biomarkers in cancer. Dis Markers 2017: 7243968, 2017.

38. Luo J, Qu J, Wu DK, Lu ZL, Sun YS and Qu Q: Long non-coding RNAs: A rising biotarget in colorectal cancer. Oncotarget 8: 22187-22202, 2017.

39. Fatima R, Akhade VS, Pal D and Rao SM: Long noncoding RNAs in development and cancer: Potential biomarkers and therapeutic targets. Mol Cell Ther 3: 5, 2015.
40. Huang S, Qing C, Huang Z and Zhu Y: The long non-coding RNA CCAT2 is up-regulated in ovarian cancer and associated with poor prognosis. Diagn Pathol 11: 49, 2016

41. Nikpayam E, Tasharrofi B, Sarrafzadeh S and Ghafouri-Fard S: The role of long non-coding RNAs in ovarian cancer. Iran Biomed J 21: 3-15, 2017.

42. Tang Q, Ni Z, Cheng Z, Xu J, Yu H and Yin P: Three circulating long non-coding RNAs act as biomarkers for predicting NSCLC. Cell Physiol Biochem 37: 1002-1009, 2015.

(c) (i) () This work is licensed under a Creative Commons Attribution-NonCommercial-NoDerivatives 4.0 International (CC BY-NC-ND 4.0) License. 